... Katrin Raess, Fachärztin für Urologie (1998 die erste in der Schweiz)

\title{
«Ich bin es gewohnt, ungewöhnliche Fragen zu stellen»
}

\section{Daniel Lüthi}

Text und Bilder
Auf dem Tisch im Wartezimmer liegen weder Männer- noch Frauenmagazine, sondern Bücher. Humoristische von Loriot und Busch etwa - oder der «Struwwelpeter», geschrieben vom Arzt Heinrich Hoffmann. Eine spezielle Geschichte, wie jedes Kind weiss. «Ich mag keine blöden Heftlein», sagt die Hausherrin zum Sortiment. Lieber lache sie über wirklich Blödes. Eine Postkarte im Sprechzimmer illustriert das: «so doof» steht darauf. Einmal habe ihr Lachen sogar Eingang in eine Todesanzeige ge- funden, ergänzt sie. In ihrer Praxis sei es immer so lustig gewesen, habe der Verstorbene der Nachwelt überliefern wollen.

\section{Männer- und Frauenärztin}

Dabei sind es doch ganz und gar ernste Themen, die in dieser Praxis mit Blick auf den Berner Zeitglockenturm diskutiert werden. Themen auch, die mit dem Ende der eigenen Zeit zu tun haben können; Nieren-, Blasen- und Prostata-Krebs zum Beispiel. Oder

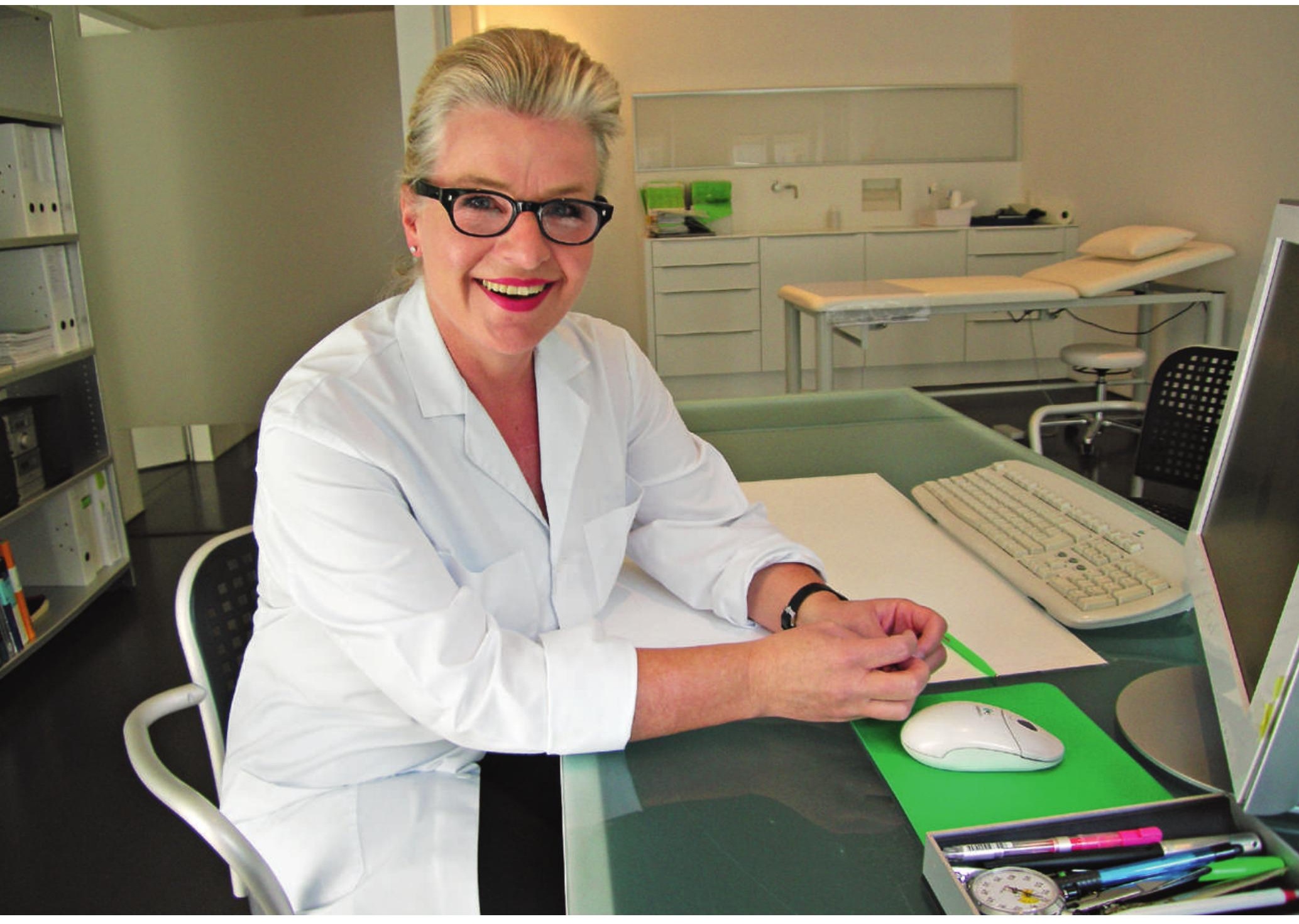


Probleme mit den Genitalien - und darum herum. Entgegen allen Klischees stehen Fragen rund um die Sexualität hier jedoch nicht im Vordergrund. «Mein grosses Hauptthema sind Harnwegs-Infekte bei jungen, sexuell aktiven Frauen», erklärt Katrin Raess. Warum? «Bei mir erhalten Frauen, die davon betroffen sind, eine ausführliche Beratung, also detaillierte Informationen über Ursachen, genetische Disposition und Mikrobiologie, dazu neuste Studienergebnisse. Ich sehe sie eine Stunde lang, dafür kommen die Frauen dann während langer Zeit ganz gut selbst zurecht, können auf Notfallkonsultationen während der Nacht oder am Wochenende verzichten.» Dreieinhalb Tage pro Woche widme sie ihren Sprechstunden in der Praxis. «Einen halben Tag verbringe ich mit transurethralen Eingriffen und einen ganzen Tag mit schnittchirurgischen Eingriffen im Spital. Alle paar Wochen sind an einem Samstag Haus- und Heimbesuche angesagt. Es leuchtet mir nicht ein, wieso sich die zum Teil schwerstbehinderten Patienten für einfache urologische Behandlungen in die Praxis begeben müssen.»

Vor ein paar Jahren noch wurde Raess als «Männerärztin» bezeichnet. Das wäre jetzt nicht mehr

\section{«Schamgefühle gehören dazu.»}

korrekt. «Heute besteht mein Patientengut zu 50 bis 60 Prozent aus Frauen. Bei männlichen Kollegen liegt der weibliche Anteil bei nur 5 bis 10 Prozent.» Mit anderen Worten: Frauen gehen lieber zu Frauen und Männer zu Männern, wenn es um besonders Intimes geht. Raess selber sagt es so: «Der Markt hat mich gefunden.»

Viele Männer, bei denen es um die äusseren Genitalien, um Erektionen und Potenz geht, kommen auch hierher. Fast alle Patienten seien nett, aufrichtig, differenziert, manche hätten vielleicht Hemmungen: «Schamgefühle gehören dazu.» Offenbar auch bei Kollegen. Deshalb wohl würden ihr Patienten immer wieder die gleiche Geschichte erzählen: «Vom Zuweiser werden sie gefragt, ob es ihnen gleich sei, wenn er sie zu einer Frau schicke. Statt dass er sie fragt, ob er sich lieber von einem Mann oder von einer Frau behandeln lassen will. Obschon: Wenn einer sein Ohr oder sein Herz zeigen muss, ist das ja auch keine Frage.»

\section{Frau in einem Männerberuf}

Warum hat Katrin Raess damals, Ende der achtziger Jahre, als erste Frau in der Schweiz Urologin werden und sich eben nicht um Ohren oder Herzen kümmern wollen? «Da war ein Zufall dahinter, dann eine grosse Bewunderung für meinen Chef, aber wahrscheinlich auch etwas aus meiner frühen Kindheit in

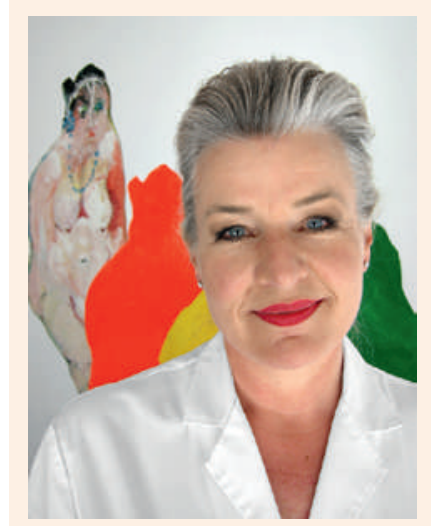

\section{Katrin Raess}

Dr. med. Katrin Raess wurde 1960 in Bern geboren, wo sie auch die Schulen besuchte. Medizin studierte sie in Bern sowie in Washington DC und Rochester NY in den USA. Nach dem Staatsexamen 1986 bildete sie sich weiter in Innerer Medizin (Bern und Moutier), Chirurgie (Basel) Gynäkologie und Geburtshilfe (Olten) und Anästhesie (Biel).

1994 wurde sie Fachärztin für Allgemeine Medizin, 1998 - als erste Frau in der Schweiz - Fachärztin für Urologie. 1999 eröffnete sie in Bern ihre eigene Praxis. Zudem ist sie Belegärztin am Berner Salemspital. Zusammen mit ihrem Partner lebt Katrin Raess in Bern und Luzern.

den 60er Jahren. Meine Mutter hatte alles daran gesetzt, aus mir ein liebenswertes, dienendes, manierliches Elfchen zu machen. Ich wollte zwar nie ein Bub sein, fand aber die Welt der Knaben immer viel spannender als diejenige der Mädchen, ich wollte eine unter ihnen sein. Die Vorliebe für ein männliches Setting ist mir geblieben. Etwas in einer reinen Frauengruppe zu unternehmen, beklemmt mich immer noch, an einem Ladies-Lunch teilzunehmen, finde ich fürchterlich, und wenn dann zum $\mathrm{Ab}$ schluss noch kleine Kosmetiktäschchen verteilt werden, möchte ich weinen.»

Der erwähnte Zufall hat damit zu tun, dass in der Weiterbildungszeit einst eine Stelle in der Inneren Medizin im letzten Moment abgesagt wurde und die Urologie geografisch gleich nebenan lag. Dass Katrin Raess Urologin wurde, hat aber auch damit zu tun, dass es ihr im anderen damals typisch männlichen Fach, der Gynäkologie, ganz und gar nicht gefiel. «Der Gyni-Alltag hat mich deprimiert. Das Abtreibungsscreening und die Unterbrechungscurettagen setzten mir besonders zu. Nach einem Jahr wollte ich zurück auf die Urologie.» 


\section{Männer- und Frauenwelten}

Mit dieser Wahl war Katrin Raess also für eine eidgenössische Premiere besorgt. Heute noch gehört sie übrigens zu einer kleinen Minderheit, von rund 220 aktiven Urologen in der Schweiz sind nur 10 Frauen. «Besonders am Anfang war ich immer wieder mit unangenehmen Witzeleien konfrontiert. Aber die perlten an mir ab wie Duschwasser, da bin ich zu selbstbewusst.» Auch deshalb übrigens fühlt sie sich mehr in männliche als in weibliche Welten gezogen. «Mit Männern komme ich schneller zu einem Punkt. Sie entscheiden selber, sehen sich nicht gleich als Opfer. Frauen müssen oft zuerst ihren Partner oder eine Freundin fragen, ob sie wirklich eine Therapie machen sollen. Manchmal zitieren sie Frauenzeitschriften. Das ärgert mich im Innern. Männer recherchieren oft im Internet und kommen informiert und dokumentiert zur Besprechung, sie sehen die unterschiedlichen Ansichten und lassen sich beraten, wenn sie Vertrauen geschöpft haben.»

Für ihren Partner, der aus der Wirtschaft kommt sei es nicht immer einfach gewesen, sich mit ihr zusammen in einer Gesellschaft zu bewegen. «Hold lächeln und Zugemüse spielen liegt mir nicht. Lieber unterhalte ich mich mit Männern. Bloss: Die sollen mit anderem glänzen als mit Urologenwitzen!»

Erektile Dysfunktionen, also Potenzstörungen, stehen bei ihren männlichen Patienten - allen Klischees zum Trotz - eher im Hintergrund. Und wenn schon: «Diese haben eher mit gesundheitlichen Problemen als mit gesellschaftlichen Phänomenen im Sinne von Lifestyle zu tun. Hauptrisikofaktor ist das Rauchen. Der Schwellkörper kann Indikatororgan einer beginnenden kardiovaskulären Erkrankung sein.»

Gutartige Vergrösserungen der Prostata, Blasenentzündungen oder Nierentumore sind alltäglicher. Und: «Sexuell übertragbare Krankheiten nehmen zu. Am häufigsten sehe ich die Chlamydien-Urethritis.»

Und wie steht es mit Konsultationen, die in den Bereich «Lifestyle» gehören? «Natürlich gibt es Männer, die sich stundenlang Bilder von Genitalien ansehen und dann mit genauen Vorstellungen kommen, wie ihr Penis aussehen sollte. Ich denke aber, dass die meisten, die einen plastischen Eingriff wünschen, sich im Ausland operieren lassen.» Pause. «Komplikationen von Piercings sehe ich selten.» Pause. «Ab und zu kommt einer, der seinen Termin bei der Ärztin als Event sieht, der endlich einmal mit einer Frau über etwas reden will, das er sonst niemandem sagen kann.»

Gemeinsamer Nenner im Praxis-Alltag von Katrin Raess: Direktheit. «Ich bin es gewohnt, ungewöhnliche Fragen zu stellen. Und zu beantworten.»

\section{Reduktion als Luxus}

So verheimlicht sie auch gewisse Ermüdungserscheinungen nicht. "Oft habe ich den Eindruck, langsam gesättigt zu sein. Ich merke, dass ich zunehmend mehr Ferien brauche. 12 Wochen pro Jahr sind es zurzeit. Weniger arbeiten: Das ist mein Luxus. Und meine Praxisassistentin profitiert natürlich auch davon.»

Apropos «Luxus»: Urologen haben den Ruf, unter den Ärzten zur Spezies der Grossverdiener zu gehören. «Ich verdiene sicher bloss die Hälfte von dem, was meine Kollegen nach Hause tragen», sagt Raess dazu, «wegen des hohen Frauenanteils und der Krankheitsbilder, die hauptsächlich eine Beratung verlangen, generiere ich weniger Operationsvolumen.» Für das, was sie sich leisten wolle, reiche ihr Einkommen allerdings bestens. «Ich reise sehr

\section{«Hold lächeln und Zugemüse spielen liegt mir nicht.»}

gerne, oft in den Vorderen Orient, zu meiner Schwester nach Israel zum Beispiel. Oder zu einem ihrer 6 Kinder und deren Familien, die über die ganze Welt verteilt sind. Das sind alles eigenwillige, lustige Menschen. Und ja: Ich habe gerne edle Rückzugsmöglichkeiten, auch in den Bergen, im Bergell zum Beispiel. Ich übernachte also nicht auf dem Campingplatz. Auch koche und esse ich sehr gern.»

Hier, beim Reisen und beim Essen, wird allerdings auch ein anderes Charakteristikum von Katrin Raess deutlich. «Ich liebe die Reduktion. Auch beim Geschmack. Deshalb gefällt mir die einfache, klare italienische Küche. Und deshalb ist Japan das Land meiner Träume. Diese Ästhetik, diese extreme Reduktion faszinieren mich.»

Übrigens: Sie sei nicht die Frau der hundert Handtaschen, besitze nur eine Uhr und ein Paar Diamanten im Ohr, sagt sie. Und ihre Wohnung sei, verglichen mit ihrem Elternhaus beispielsweise, praktisch leer. Im Vergleich dazu sei ihre Praxis eher übermöbliert.

Die Reduktion als Kompass. Urologin unter lauter Urologen - und trotzdem kein Privatflugzeug und kein Segelboot. Frau - und trotzdem keine Kinder und kein Ehemann. «Allen Ernstes wurde ich von durchaus intelligenten Menschen auch schon als randständig bezeichnet.» Sagt's - und lacht. Und nimmt's als Kompliment.

\section{Die nächste «Begegnung mit ...»}

Am Ende jeden Monats stellt die Schweizerische Ärztezeitung eine Persönlichkeit vor, die sich im Gesundheitswesen engagiert. Im Juni schildert Daniel Lüthi seine Begegnung mit Prof. Peter MeierAbt, Internist, Klinischer Pharmakologe und Präsident der Schweizerischen Akademie der Medizinischen Wissenschaften SAMW. 